\title{
ADVANCES IN TRANSCATHETER AORTIC VALVE REPLACEMENT
}

\author{
Neal S. Kleiman, M.D.; Michael J. Reardon, M.D. \\ Houston Methodist DeBakey Heart \& Vascular Center, Houston Methodist Hospital, Houston, Texas
}

\begin{abstract}
Transcatheter aortic valve replacement (TAVR) is becoming widely used for the treatment of symptomatic severe aortic stenosis in patients with high surgical risk. Data from The PARTNER Trial (Placement of AoRtic TraNscathetER Valves) and the Medtronic

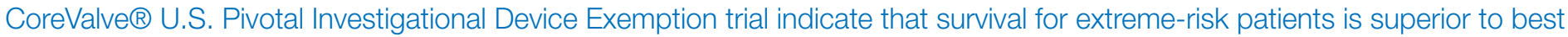
medical therapy and equivalent or superior to surgical aortic valve replacement (SAVR), although long-term durability remains unknown. Paravalvular leak remains higher in TAVR than SAVR, as does permanent pacemaker implantation in self-expanding valves. New-generation valves are addressing these issues, especially for paravalvular leak. There is strong evidence that TAVR is appropriate for both extreme-risk and high-risk patients with symptomatic severe aortic stenosis, and the continued development of new valves are making implantation more reliable. This review discusses the studies supporting the use of TAVR and explores current advances in the field.
\end{abstract}

\section{Introduction}

Degenerative aortic stenosis (AS) is the most common cardiac valve disease requiring surgical treatment in developed countries and increases in incidence as the population ages. ${ }^{1}$ Survival without treatment is very poor in symptomatic patients, approaching $50 \%$ at 1 year in the highest risk groups and in those with symptomatic severe AS. ${ }^{2}$ Until recently, the only therapy shown to prolong life and relieve symptoms was surgical aortic valve replacement (SAVR). ${ }^{3}$ However, as many as half of those who met the guideline recommendations for SAVR had been denied or refused treatment based on age, frailty, comorbidities, or personal choice. ${ }^{4}$ Transcatheter aortic valve replacement (TAVR) was developed to offer this high-risk population a less-invasive alternative to SAVR. There are currently two families of TAVR valves that are commercially available and approved by the U.S. Food and Drug administration (FDA) for the treatment of symptomatic severe AS in extreme and high-risk patients based on their U.S. investigational device exemption (IDE) trials. These are the balloon-expandable SAPIEN series (Edwards Lifesciences Corporation, Irvine, CA), consisting of the SAPIEN valve (Figure 1, no longer available), SAPIEN XT valve, and the newest generation SAPIEN 3 valve, and the self-expanding CoreValve ${ }^{\circledR}$ System (Medtronic, Inc., Minneapolis, MN), composed of the CoreValve and the new generation Evolut ${ }^{\circledR} R$ valves. This review examines the evidence that led to the approval of TAVR for extreme ("inoperable") and for high-risk patients and discusses recent advances as well as the current state of TAVR.

\section{Extreme-Risk Patients}

The FDA approval of the Sapien and CoreValve systems was based on seminal U.S. IDE trials that included extreme or inoperable as well as high-risk cohorts for each valve. The PARTNER B trial (Placement of AoRtic TraNscathetER Valves) was a randomized noninferiority study that compared TAVR using the Sapien valve against conservative or best medical therapy (BMT) in the treatment of symptomatic patients with severe AS. At 1 year there was a $20 \%$ absolute survival advantage for TAVR over medical therapy, a rate that met noninferiority criteria and led to the approval of this valve for patients deemed to be inoperable. ${ }^{2}$
The cohorts have been followed to the trial's 5-year end point, and a $20 \%$ survival advantage for TAVR remains. Notably, among patients randomized to BMT, there was only one survivor at this point who did not ultimately require a valve replacement. ${ }^{5}$ The Medtronic CoreValve ${ }^{\circledR}$ U.S. Pivotal IDE trial also used a noninferiority design to compare TAVR using the CoreValve system to an objective performance goal (43\%) that was derived from the observed mortality in the BMT arm of PARTNER B and five contemporary balloon aortic valvuloplasty series. The 1-year death or major stroke rate in the CoreValve IDE trial was $26 \%$, and all-cause mortality was $23.7 \%$. The latter value was significantly different than the performance goal ${ }^{6}$ and led to rapid FDA approval in extreme risk patients. ${ }^{6}$ This trial has been followed out to 2 years with a mortality of $36 \%{ }^{7}$

\section{High-Risk Patients}

The PARTNER A investigators studied patients who were considered a high risk for surgery. The Trial used a noninferiority design with a primary end point of all-cause mortality at 1 year. Patients were randomized to either TAVR using the Sapien valve or SAVR. The 1 -year mortality was $24.2 \%$ vs $26.8 \%$ for TAVR vs SAVR, which met noninferiority criteria ${ }^{8}$ and led to FDA approval of this valve in high-risk patients. Of note in this early trial, the rate of all stroke was significantly greater in TAVR vs SAVR at 1 year $(8.3 \%$ vs $4.3 \%, P=0.04)$, which raised early concerns. This trial has been followed to its 5-year end point with a mortality of $67.8 \%$ vs $62.4 \%$ for TAVR vs SAVR, respectively, maintaining noninferiority. ${ }^{9}$ Also of note is the fact that stroke was no longer different in the groups at either the 3-year or the 5-year end point. The CoreValve high-risk trial also randomized TAVR vs SAVR in symptomatic high-risk patients with severe AS, with a primary end point of all-cause mortality at 1 year. This study was designed as a noninferiority trial with a prespecified hierarchical superiority test if noninferiority was reached. The mortality at 1 year was $14.2 \%$ for TAVR vs $19.1 \%$ for SAVR, which met noninferiority and superiority criteria $(P=0.04)$ and led to FDA approval. ${ }^{10}$ This trial was the first and so far the only randomized trial to ever show superiority for TAVR vs SAVR. In contrast to the PARTNER A trial, stroke occurred in $12.6 \%$ for SAVR vs $8.8 \%$ for TAVR, and 


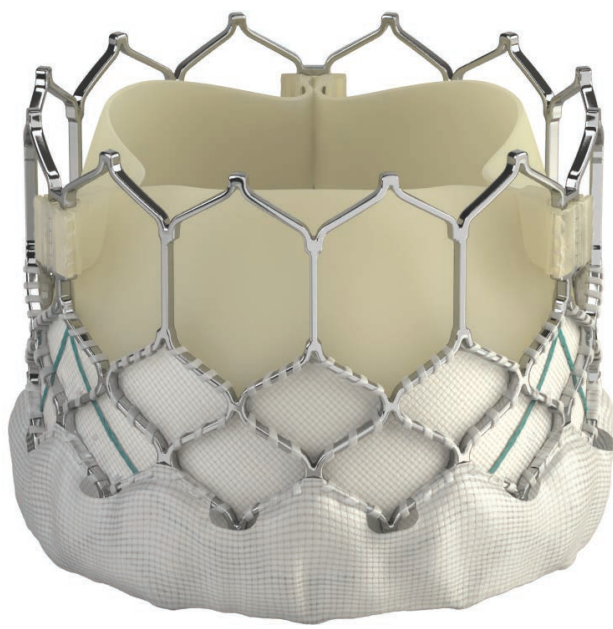

Figure 1. Sapien 3 valve, used with permission by Edwards Lifesciences.

the rates were not different $(P=0.10)$, helping to alleviate some of the concerns about stroke with TAVR. After 2 years of follow-up, TAVR in the CoreValve trial showed superiority in mortality for TAVR vs SAVR $(22.2 \%$ vs $28.6 \%, P=0.04)$ as well as a strong trend in favor of TAVR for stroke rates $(10.9 \%$ vs $16.6 \%, P=0.05) .{ }^{11}$

Several other outcomes from this set of trials impact TAVR's standing. The improvement in quality of life (QoL) was very large in the extreme-risk trials ${ }^{12,13}$ and in the randomized trials at 1 year was equal between TAVR and SAVR, although transfemoral TAVR had a more rapid attainment of this improved QoL. ${ }^{14,15}$ The hemodynamic flow parameters in the randomized trials showed equivalence or superiority to surgical valves. ${ }^{9,11}$ Paravalvular leak (PVL), however, was less common after SAVR in all trials. The need for a permanent pacemaker (PPM) in TAVR was equivalent to SAVR in PARTNER A and inferior to SAVR in the CoreValve high-risk group, although the need for a PPM in the CoreValve trials was not associated with increased mortality, and this reflects the TAVR literature in general. Although rare, occasional malposition of either valve with regard to the aortic annulus required placement of an additional valve in the proper position. Consequently, these observations leave TAVR with several challenges, including overcoming PVL and the need for PPM and ensuring accurate placement every time, particularly as indications are expanded to lower-risk and younger patients with longer expected survival. In particular, TAVR in this population adds the requirement of durability.

\section{Solving the Problems}

Paravalvular leak has consistently been less common after SAVR than TAVR using any valve. The causes of PVL include a valve that is too small, a malpositioned valve (too high or low) with respect to the aortic annulus, and the patient's anatomic factors such as extensive or eccentric calcification of the annulus, which leads to imperfect sealing of the space between the valve and annulus. Choosing the correctly sized valve has been greatly improved with the use of 3-dimensional (3D) computed tomography angiography imaging as opposed to $2 \mathrm{D}$ echo imaging for critical measurements. This problem has been further addressed with the development of completely recapturable valves that can be exchanged for larger valves if needed. Malposition is not a common problem but should be eliminated by the current generation of self-expanding and expandable valves that allow recapture, reposition, and redeployment before release. The final issue of patient anatomy is more complex. Repositionability will help by allowing the implanting physician to choose the optimal landing site for the valve, which is not always obvious on the first attempted deployment. Additionally, valves have been developed with sealing skirts to fill the uneven spots along the landing zone, which helps to eliminate PVL.

The need for permanent pacemaker implantation has been higher in the self-expanding valves than in the balloon-expandable valves. With self-expanding valves, the depth of implantation and possibly "oversizing" the valve leading to stretching of the left ventricular outflow tract and conduction system have been directly correlated with the need for PPI. Valve length may also play a role in increasing the force at the level of the conduction tissue.

\section{The New Commercially Available Valves}

The SAPIEN 3 (S3) valve is the newest member of the SAPIEN family. It has a balloon-expandable cobalt chromium frame with a bovine pericardial valve mounted in the frame. Compared to the previous-generation SAPIEN XT, the S3 can be inserted thru smaller iliofemoral arteries, has an improved delivery system that allows more accurate positioning, and contains a skirt to help prevent PVL. The 30-day data presented by Susheel Kodali at the American College of Cardiology 2015 meeting showed a moderate to severe PVL rate of $3.8 \%$ and PPI rate of $13 \% .{ }^{16}$ Tarantini has published 30-day results of S3 implantation in 209 European patients showing a moderate to severe PVL rate of $0 \%$ and PPM rate of $20.68 \% .{ }^{17}$ This valve is currently approved in the United States for high- and extreme-risk patients with symptomatic severe AS.

The Medtronic Evolut $\mathrm{R}$ is the new generation of the CoreValve self-expanding family of valves (Figure 4). The valve has been re-engineered to be shorter, to provide a more consistent radial force within the recommended size range, and to allow recapture, repositioning, and redeployment prior to final release. The 30-day data was presented at Euro PCR by Ian Meredith and showed a moderate to severe PVL rate of $3.4 \%$ and PPI of $12.4 \%{ }^{18}$ This valve is currently approved in the United States for high- and extremerisk patients with symptomatic severe AS.

\section{The New Trial Valves}

The Lotus ${ }^{\mathrm{TM}}$ valve (Boston Scientific, Marlborough, MA) is a partially self-expanding and then expandable valve (Figure 2).

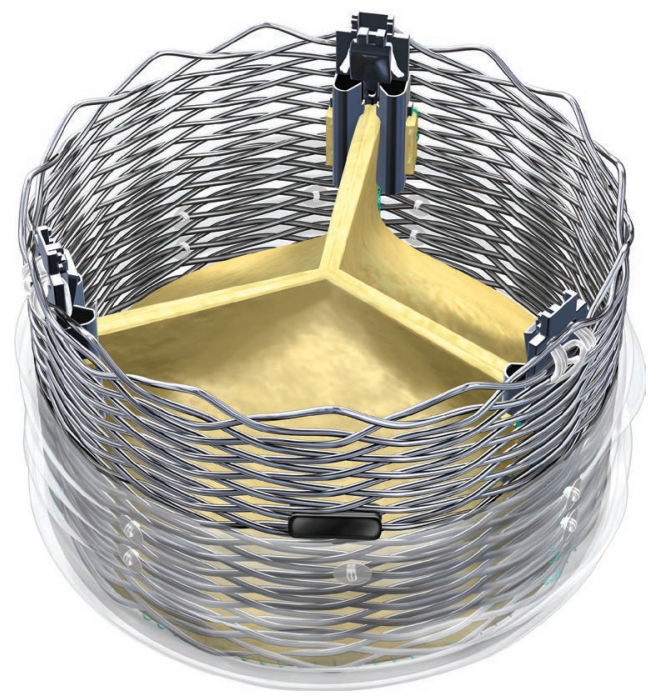

Figure 2. Lotus ${ }^{\mathrm{TM}}$ valve, used with permission by Boston Scientific. 
It has a nitinol frame with a bovine pericardial valve mounted inside. In addition, the top and bottom of the nitinol frame have a buckle and post system connected by a wire. As the delivery system handle is rotated, it brings these components together to shorten and widen the valve. The valve is never obstructive at any point in the deployment, allowing optimal hemodynamic stability. This allows the valve to be "dialed" into position with a radial force that is greater than a self-expanding valve alone but less than a balloon-expandable valve. The Lotus valve can be fully deployed to the lock position and then fully evaluated. The implanter can then decide to release the valve if all looks good or recapture, reposition, and redeploy it to attain a better outcome. If necessary, the valve can also be completely removed and a different (larger or smaller) valve deployed to obtain optimal results. The 30-day results in the Reprise II study showed a 1\% moderate and no severe PVL rate. ${ }^{19}$ The PPM rate was $28.6 \%$ but the trial included only two valve sizes, $23 \mathrm{~mm}$ and $27 \mathrm{~mm}$. As a result, patients in the middle of this range often received an oversized $27-\mathrm{mm}$ valve, which is thought to have contributed to the PPM rate. The U.S. IDE Reprise III trial is testing 23-mm, 25-mm, and 27-mm valves.

The Direct Flow Medical ${ }^{\circledR}$ valve (Direct Flow Medical, Inc., Lake Forest, CA) is a new valve constructed of hollow Dacron tubes that provide the valve skeleton (Figure 3 ). When inflated, the tubes create a rigid scaffold with a bovine pericardial valve mounted inside. The valve has a ventricular ring that conforms to the annulus and an aortic ring that opens the top of the valve to push the old leaflets out of the way. It is positioned in the left ventricular outflow tract and is expanded by filling the Dacron skeleton with a saline contrast mixture. The aortic ring is deflated, which allows the valve to be manipulated into position until the ventricular ring is properly aligned with the annulus. The aortic ring is then inflated to bring the valve to its final shape. The valve is functional during this entire time, allowing stable hemodynamics, and can be fully assessed at full deployment. If the implant is not optimal, the valve can be deflated for repositioning or exchange. If the implant is optimal, then the saline contrast mix is exchanged for a quick-setting polymer that transforms into the valve's permanent shape. In the first study performed, aortic regurgitation was mild or less in $98.6 \%$ of patients. ${ }^{20}$ Both the Lotus and Direct Flow Medical valves are commercially available in Europe but remain investigational in local and national U.S. research centers.

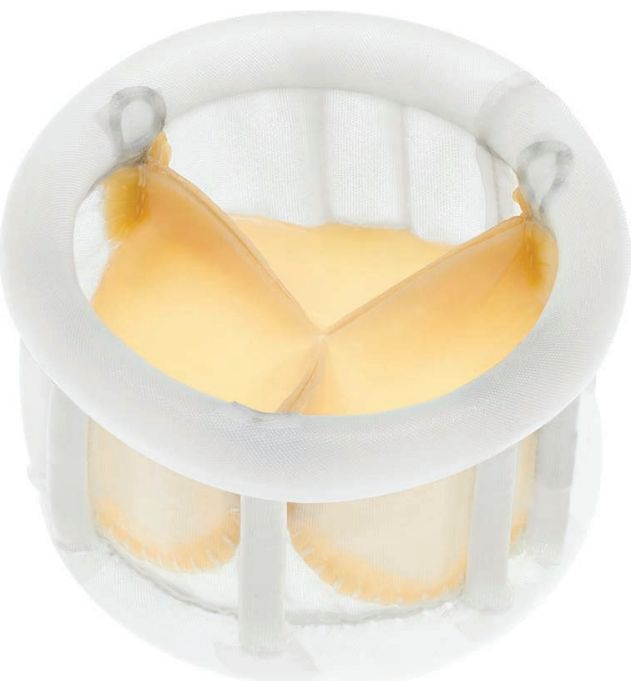

Figure 3. The Direct Flow Medical ${ }^{\circledR}$ Transcatheter Aortic Valve, used with permission by Direct Flow Medical.

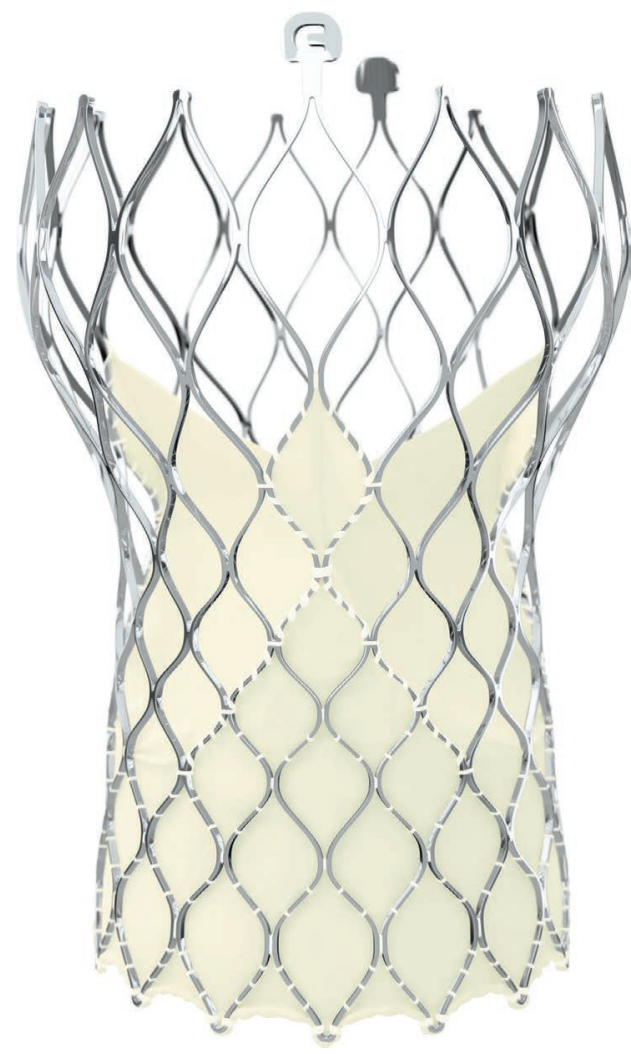

Figure 4. Evolut@ R valve, used with permission by Medtronic (C2015.

Durability remains a question for all of these devices as all currently used valves have been employed for less than a decade. True durability data is unlikely until younger, lower-risk populations are treated and will likely need another 8 to 10 years for a reasonable answer.

\section{Intermediate Risk}

Intermediate-risk patients are currently being treated with TAVR in Europe, and early data suggests survival is equivalent to surgery. The definitive answer will likely await the results of the PARTNER II A and SURTAVI (Surgical Replacement and Transcatheter Aortic Valve Implantation) randomized intermediate-risk trials, both of which have 2-year survival as primary end points. PARTNER II A has met its enrollment goal and 2-year data should be available later this year. The SURTAVI trial is being conducted by the Houston Methodist Hospital and is expected to complete enrollment by the end of 2015 .

\section{Conclusion}

TAVR has become an important component in our ability to treat aortic valve disease. The steady improvement of existing valves and development of new novel valves has allowed continued expansion of this area. In 2014 at the Houston Methodist Hospital, slightly more than $30 \%$ of our isolated aortic valve replacement cases were done as TAVR rather than open surgical replacement. We would expect that number to continue to increase and patient outcomes to continue to improve as the field matures.

Conflict of Interest Disclosure: Dr. Reardon serves on the scientific advisory board for Medtronic, Inc.

Keywords: transcatheter aortic valve replacement, TAVR, severe aortic stenosis, aortic valve, paravalvular leak, permanent pacemaker implantation 


\section{References}

1. Carabello BA, Paulus WJ. Aortic stenosis. Lancet. 2009 Mar 14;373(9667):956-66.

2. Leon MB, Smith CR, Mack M, et al.; PARTNER Trial Investigators. Transcatheter aortic-valve implantation for aortic stenosis in patients who cannot undergo surgery. N Engl J Med. 2010 Oct 21;363(17):1597-607.

3. Bonow RO, Carabello BA, Chatterjee K, et al.; American College of Cardiology/American Heart Association Task Force on Practice Guidelines. ACC/AHA 2006 Guidelines for the Management of Patients with Valvular Heart Disease: a Report of the American College of Cardiology/American Heart Association Task Force on Practice Guidelines (Writing Committee to Revise the 1998 Guidelines for the Management of Patients With Valvular Heart Disease). Developed in Collaboration with the Society of Cardiovascular Anesthesiologists; Endorsed by the Society for Cardiovascular Angiography and Interventions and the Society of Thoracic Surgeons. Circulation. 2006;114(5):e84231.

4. lung B, Cachier A, Baron G, et al. Decision-making in elderly patients with severe aortic stenosis: why are so many denied surgery? Eur Heart J. 2005 Dec;26(24):2714-20.

5. Kapadia SR, Leon MB, Makkar RR, et al.; PARTNER trial investigators. 5-year outcomes of transcatheter aortic valve replacement compared with standard treatment for patients with inoperable aortic stenosis (PARTNER 1): a randomised controlled trial. Lancet. 2015 Jun 20;385(9986):2485-91.

6. Popma JJ, Adams DH, Reardon MJ, et al.; CoreValve United States Clinical Investigators. Transcatheter aortic valve replacement using a self-expanding bioprosthesis in patients with severe aortic stenosis at extreme risk for surgery. J Am Coll Cardiol. 2014 May 20;63(19):1972-81.

7. Yakubov SJ, Adams DH, Watson DR, et al.; CoreValve United States Clinical Investigators. 2-Year Outcomes After lliofemoral Self-Expanding Transcatheter Aortic Valve Replacement in Patients with Severe Aortic Stenosis Deemed Extreme Risk for Surgery. J Am Coll Cardiol. 2015 Sep 22;66(12):1327-34.

8. Smith CR, Leon MB, Mack MJ, et al.; PARTNER Trial Investigators. Transcatheter versus surgical aortic-valve replacement in high-risk patients. N Engl J Med. 2011 Jun 9;364(23):2187-98.

9. Mack MJ, Leon MB, Smith CR, et al.; PARTNER 1 trial investigators. 5-year outcomes of transcatheter aortic valve replacement or surgical aortic valve replacement for high surgical risk patients with aortic stenosis (PARTNER 1): a randomised controlled trial. Lancet. 2015 Jun 20;385(9986):2477-84.
10. Adams DH, Popma JJ, Reardon MJ. Transcatheter aortic-valve replacement with a self-expanding prosthesis. N Engl J Med. 2014 Sep 4;371(10):967-8.

11. Reardon MJ, Adams DH, Kleiman NS, et al. 2-Year Outcomes in Patients Undergoing Surgical or Self-Expanding Transcatheter Aortic Valve Replacement. J Am Coll Cardiol. 2015 Jul 14;66(2):113-21.

12. Reynolds MR, Magnuson EA, Lei Y, et al.; Placement of Aortic Transcatheter Valves (PARTNER) Investigators. Health-related quality of life after transcatheter aortic valve replacement in inoperable patients with severe aortic stenosis. Circulation. 2011 Nov 1;124(18):1964-72.

13. Osnabrugge RL, Arnold SV2, Reynolds MR, et al.; CoreValve U.S. Trial Investigators. Health status after transcatheter aortic valve replacement in patients at extreme surgical risk: results from the CoreValve U.S. trial. JACC Cardiovasc Interv. 2015 Feb;8(2):315-23.

14. Reynolds MR, Magnuson EA, Wang K, et al.; PARTNER Trial Investigators. Health-related quality of life after transcatheter or surgical aortic valve replacement in high-risk patients with severe aortic stenosis: results from the PARTNER (Placement of AoRTic TraNscathetER Valve) Trial (Cohort A). J Am Coll Cardiol. 2012 Aug 7;60(6):548-58.

15. Arnold SV, Reynolds MR, Wang K, et al.; CoreValve US Pivotal Trial Investigators. Health Status After Transcatheter or Surgical Aortic Valve Replacement in Patients With Severe Aortic Stenosis at Increased Surgical Risk: Results From the CoreValve US Pivotal Trial. JACC Cardiovasc Interv. 2015 Aug 17;8(9):1207-17.

16. Kodali S. Early clinical and echocardiographic outcomes with the SAPIEN 3 transcatheter aortic valve replacement system in inoperable, high-risk and intermediate-risk aortic stenosis patients. Research presented at: American College of Cardiology 2015 Scientific Sessions; 2015 Mar 15; San Diego, CA.

17. Tarantini G, Mojoli M, Purita P, et al. Unravelling the (arte)fact of increased pacemaker rate with the Edwards SAPIEN 3 valve. Eurolntervention. 2015 Jul;11(3):343-50.

18. Manoharan G, Walton AS, Brecker SJ, et al. Treatment of Symptomatic Severe Aortic Stenosis With a Novel Resheathable Supra-Annular Self-Expanding Transcatheter Aortic Valve System. JACC Cardiovasc Interv. 2015 Aug 24;8(10):1359-67.

19. Meredith IT, Worthley SG, Whitbourn RJ, et al. Transfemoral aortic valve replacement with the repositionable Lotus Valve System in high surgical risk patients: the REPRISE I study. Eurolntervention. 2014 Mar 20;9(11):1264-70.

20. Schofer J, Colombo A, Klugmann S, et al. Prospective multicenter evaluation of the direct flow medical transcatheter aortic valve. J Am Coll Cardiol. 2014 Mar 4;63(8):763-8. 